\title{
Investigation of Flow Around a Slender Body at High Angles of Attack
}

\author{
Ibraheem AlQadi and Eltayeb ElJack \\ Aeronautical Engineering Department, Faculty of Engineering, \\ King Abdulaziz University, P.O. Box 80204, Jeddah, 21589, Saudi Arabia \\ ialqadi@kau.edu.sa
}

\begin{abstract}
A numerical investigation of flow around a slender body at high angles of attack is presented. Large eddy simulation of the flow around an ogive-cylinder body at high angles of attack is carried out. Asymmetric vortex flow was observed at angles of attack of $\alpha=55^{\circ}$ and $65^{\circ}$. The results showed that the phenomenon is present in the absence of artificial geometrical or flow perturbation. Contrary to the accepted notion that flow asymmetry is due to a convective instability, the development of vortex asymmetry in the absence of perturbations indicates the existence of absolute instability. An investigation of the unsteady flow field was carried out using dynamic mode decomposition. The analysis identified two distinct unsteady modes; high-frequency mode and low-frequency mode. At angle of attack $45^{\circ}$ the high-frequency mode is dominant in the frontal part of the body and the low-frequency mode is dominant at the rear part. At $\alpha=55^{\circ}$, the highfrequency mode is dominant downstream of vortex breakdown. At $\alpha=65^{\circ}$, the spectrum shows a wide range of modes. Reconstruction of the dynamical modes shows that the low-frequency mode is associated with the unsteady wake and the high-frequency mode is associated with unsteady shear layer.
\end{abstract}

keywords: High angle of attack aerodynamics, Flow asymmetry, Slender bodies

\section{Nomenclature}

\section{Abbreviations}

LES Large Eddy Simulation

RANS Reynolds Averaged Navier Stokes

sgs sub-grid-scale

\section{Greek Symbols}

$\alpha \quad$ angle of attack

$\Delta \quad$ spatial filter size

$\nu \quad$ kinematic viscosity

$\rho \quad$ fluid density

$\tau_{i j} \quad$ sub-grid-scale stress tensor

$\tau_{k k} \quad$ isotropic part of the sub-grid-scale stress tensor

$\nu_{t} \quad$ eddy-viscosity

\section{Roman Symbols}

$\begin{array}{ll}\mathrm{D} & \text { diameter } \\ f & \text { frequency } \\ p & \text { pressure }\end{array}$

$\mathrm{Re}_{\mathrm{D}} \quad$ Reynolds number, $\mathrm{Re}_{\mathrm{D}}=\mathrm{V}_{\infty} \mathrm{D} / \nu$

$S_{i j} \quad$ strain rate tensor

St $\quad$ Strouhal number, $\mathrm{St}=f \mathrm{D} / \mathrm{V}_{\infty}$

$u_{i} \quad$ velocity vector

$\mathrm{V}_{\infty} \quad$ freestream velocity

$x_{i} \quad$ coordinates vector

\section{Introduction}

High angles of attack flow around slender bodies is categorized into four types; a low range of symmetric flow $\left(\alpha<30^{\circ}\right)$, an intermediate range $\left(30^{\circ}<\alpha<50^{\circ}\right)$ of steady asymmetric flow, a nearly bistable mode within the range $\left(50^{\circ}<\alpha<65^{\circ}\right)$, and a vortex shedding mode in the range $\left(\alpha>65^{\circ}\right)^{[1]}$. Early experimental studies have shown that high angles of attack flow field is affected by Reynolds number, Mach number, fineness ratio, and the nose bluntness. Additionally it was found that the 
flow field is highly sensitive to small changes in testing conditions as well as geometrical irregularities due to imperfections in model constriction. The flow visualization study of Gowen et $a l .{ }^{[2]}$ investigated the effects of nose shape, shape bluntness, and Reynolds number on the onset of asymmetric vortex flow. It was found that the asymmetry onset angle of attack is increased by reducing the fineness ratio or increasing nose bluntness. Reynolds number was found to be relevant only for high fineness ratio in which increasing the Reynolds number decreases the asymmetry onset angle of attack. Wardlaw and Morrison ${ }^{[3]}$ investigated several sets of data collected from fifteen different sources. A linear regression analysis was performed to extract flow field trends. The analysis emphasized some of the finding by Gowen et $a{ }{ }^{[2]}$ such as the effect of fineness ratio and nose bluntness. However, the investigation also concluded that the asymmetry onset angle of attack decreases with increasing model length and the maximum side force decreases with increasing Mach number.

Zilliac $^{[1]}$ observed a strong dependency of the leeward-side flow field orientation on tip geometry. Leu et al. ${ }^{[4]}$ carried out experimental investigations on the flow field around a conventional sharp-nose ogive cylinder and an elliptic-tip ogive cylinder. The results showed that changes in the direction of the side force are related to changes in the asymmetry of the pressure distribution along the body. They found that the variation of the side force with the roll angle for the elliptic tip is more predictable than that for the sharp ogive tip. Flow visualization studies showed that, when the angle of attack increases, the locations of vortex asymmetry, vortex breakaway, and vortex breakdown propagate upstream toward the tip. In addition, it is found that, at $\alpha=45^{\circ}$ and $50^{\circ}$, the changes in the vortex structure with the roll angle occur in a more gradual manner; i.e., one can see a nearly symmetric flow pattern occurring between the two opposite asymmetric flow patterns. On the other hand, at $\alpha=60^{\circ}$, changes in the flow pat- tern with the roll angle are more abrupt, leading to the formation of a bi-stable state.

Banks et al. ${ }^{[5]}$ carried out detailed wind tunnel and flight investigations on F/A-18 configuration to investigate the causes of various high angle of attack phenomena. It was found that fixing forebody cross flow transition on model provided data that accurately match flow fields and aerodynamic characteristics of flight at high angles of attack. Wind tunnel results showed that small geometry differences especially nose boom can have a pronounced effect at high angles of attack. These differences must be modeled in sub-scale tests in order to obtain accurate correlation with flight data.

Bridges $^{[6]}$, in a review paper suggested two causes for high angles of attack vortex asymmetry; inviscid hydrodynamic instability of symmetrically separated vortices or asymmetric boundary layer separation on each side of the body. Stahl and Asghar ${ }^{[7]}$ experimentally studied the asymmetric vortex flow on two circular cones with different sizes at high angles of attack to determine the onset angle of attack of asymmetric vortex as a function of freestream Reynolds number. The two cone models have the same fineness ratio of 3.6 and the same semi apex angle of 4 degree. The Reynolds numbers were $\operatorname{Re}_{\mathrm{D}}=5 \times 10^{3}$ and $2 \times 10^{5}$ based on the bottom diameter of the cones respectively. The vortex asymmetry was determined by a smoke flow and laser-light sheet. The results showed that the onset angle of attack of asymmetric vortex decreased with increasing Reynolds numbers. Liu and Deng ${ }^{[8]}$ carried out aerodynamic force measurements and wake flow visualizations over slender bodies (cone cylinder, elliptic-nose cylinder). The experimental results showed that at angles of attack $\left(30^{\circ}-40^{\circ}\right)$, the flow is unsteady and the signal of side forces contains wide ranges of amplitudes and frequencies. The unsteadiness phenomena include low-frequency threedimensional effects, asymmetry related vortex flipping, moderate frequency von Karman vor- 
tex shedding and higher shear layer fluctuations.

The challenge of controlling the vortical flow stems from the highly unstable and perceptive nature of the flow field. Leu et al. ${ }^{[4]}$ used micro fabricated balloon actuators for side force control on a slender body. The Balloon actuators can be packaged on curve surfaces of a cone-cylinder slender body and actuated at different roll angles. Aerodynamic force measurement results indicate the effects of micro balloon actuators vary at different actuation locations on the cone-cylinder slender body. Micro balloon actuators change nose shapes of the slender body which determines adversepressure-gradient values and directly influence the origin of the separation lines and characteristics of the separated vortices over the leeside surface. Xueying and Yankui ${ }^{[9]}$ tested various active and passive forebody vortex flow control techniques such as forebody strakes, helical trips, unsteady bleed, and micro blowing. However, theses techniques were not sufficiently effective. An extensive number of experimental studies were carried out in which the asymmetry was observed and studied ${ }^{[2,3,10,1,7,5,8,9,4,11]}$. However, to the authors' knowledge, all numerical investigations of asymmetric flow around slender bodies have introduced some form of perturbations into the flow field to induce asymmetry. Otherwise, the resulting flow field is symmetric. The introduced perturbations were either geometrical perturbations or flow perturbation. Other studies used conical flow assumption and obtain asymmetric flow field. Liu et $a l .{ }^{[12]}$ predicted the asymmetry phenomena by solving the unsteady thin-layer Navier-Stokes equations. However, short-duration flow disturbances were used to initiate the asymmetry. Dejani $^{[13]}$, Dejani and Levy ${ }^{[14]}$, Levy et al. ${ }^{[15]}$ and Murman $^{[16]}$ solved Thin-Layer RANS with geometrical perturbations at the nose to simulate asymmetric flow. Kandil et al. ${ }^{[17]}$ used transitional perturbations to induce flow asymmetry. Thomson and Morrison ${ }^{[18]}$ captured the flow asymmetry. However, they used an asymmet- ric numerical scheme (Maccormack). It is also worth mentioning that most numerical studies used Thin-Layer RANS assumption which breaks down at high angles of attack and therefore the full unsteady Navier-Stokes equations have to be solved instead ${ }^{[19]}$. So far, Numerical simulations have demonstrated the necessity of perturbation at the body's nose to simulate asymmetric vortex flow. This led to the conclusion that flow asymmetry is due to a convective instability. Bridges, in a review paper ${ }^{[6]}$ , supported the premise that vortex asymmetry is due to a convective hydrodynamic instability in which a disturbance introduced at body nose will convect downstream resulting in an asymmetric vortex flow. The present work investigates whether the vortex flow asymmetry could arise in the absence of geometrical or flow disturbances. LES is carried out for the case of $\operatorname{Re}_{\infty}=10,000$. The simulation is carried out for angles of attack $\alpha=45^{\circ}, 55^{\circ}$, and $65^{\circ}$. Dynamic Mode Decomposition (DMD) is applied to the resulting numerical database to study the dynamical behavior of this flow and shed some light on the asymmetric vortex flow phenomenon.

\section{Computational Methodology}

\subsection{Mathematical Model}

The spatially filtered three-dimensional time-dependent Navier-Stokes equations are solved numerically for all motions with a scale larger than the mesh size of the chosen numerical grid, while the small-scale, mainly dissipative motion is simulated by a sub-grid scale (SGS) model. The filtered equations are given by:

$$
\begin{gathered}
\frac{\partial \bar{u}_{i}}{\partial x_{i}}=0 \\
\frac{\partial \bar{u}_{i}}{\partial t}+\frac{\partial \bar{u}_{i} \bar{u}_{j}}{\partial x_{j}}=-\frac{1}{\rho} \frac{\partial \bar{p}}{\partial x_{i}}+\frac{\partial\left(2 \nu \bar{S}_{i j}\right)}{\partial x_{j}}-\frac{\partial \tau_{i j}}{\partial x_{j}}
\end{gathered}
$$

The equations are written using the compact index-notation form where $i$ and $j$ vary from 
1 to 3. $x_{i}$ are the position vector components and $t$ denotes time. $u_{i}$ are the velocity vector components, $p$ is the pressure, $\rho$ is the fluid density and $\nu$ is the kinematic molecular viscosity. The overbar denotes spatially filtered quantities. The last term in the momentum equation is the divergence of the sub-grid-scale stresses $\tau_{i j}=\overline{u_{i} u_{j}}-\bar{u}_{i} \bar{u}_{j}$. The sub-grid-scale stresses are modeled using the eddy-viscosity concept:

$$
\begin{aligned}
\tau_{i j} & =2 v_{t} \bar{S}_{i j}+\frac{1}{3} \delta_{i j} \tau_{k k} \\
\bar{S}_{i j} & =\frac{1}{2}\left(\frac{\partial \bar{u}_{i}}{\partial x_{j}}+\frac{\partial \bar{u}_{j}}{\partial x_{i}}\right)
\end{aligned}
$$

$S_{i j}$ is the strain rate tensor and $\delta_{i j}$ is the kronecker delta. The sub-grid-scale eddy viscosity $\nu_{t}$ is computed using the dynamic Smagorinsky model of Germano et al. ${ }^{[20]}$ as modified by Lilly $^{[21]}$ :

$$
\begin{aligned}
\nu_{t} & =C_{s} \Delta^{2}|\bar{S}| \\
\bar{S} & =\sqrt{2 \bar{S}_{i j} \bar{S}_{i j}} \\
\Delta & =\left(\Delta x_{1} \Delta x_{2} \Delta x_{3}\right)^{\frac{1}{3}}
\end{aligned}
$$

The coefficient $C_{s}$ is computed dynamically by applying a second filter with filter width twice the filter width $\Delta$ based on the grid spacing. The computed value of $C_{s}$ is clipped to the range $\left(0.0<C_{s}<0.23\right)$.

\subsection{Solution Method}

The computations were carried out using Ansys-Fluent. A pressure-based solver is used to solve the above equations. The solver uses the SIMPLE scheme for pressure-velocity coupling and green-gauss node-based approach for gradients evaluation. The unsteady LES runs employ a second-order bounded central spatial discretization scheme and an implicit secondorder time marching scheme. The time step is equal to 0.0001 seconds, with a maximum of 50 error-reduction sub-iterations per time-step.

\subsection{Computational Domain and Operating Conditions}

The geometry used in the current computation is an ogive-cylinder with a diameter of $\mathrm{D}=3.5 \mathrm{~cm}$. The model length is $\mathrm{L}=$ 18.5D with slenderness ratio of 3.5 and 15 for the ogive and the cylinder respectively. The computational domain is constructed such that the domain boundaries are 6.5D upstream of the model nose, 10D downstream of the model base, and $15 \mathrm{D}$ away from the model surface in the radial direction. Velocity components were specified at in-flow boundary while extrapolation was used at the out-flow boundary. Noslip condition was applied at the model surface. The density and viscosity are held constant at $1.225 \mathrm{~kg} / \mathrm{m}^{3}$, and $1.784 \times 10^{-5} \mathrm{~kg} / \mathrm{m} . \mathrm{s}$, respectively. The free-stream velocity magnitude is fixed at $4.2 \mathrm{~m} / \mathrm{s}$ to yield a free stream Reynolds number of 10,000 based on the model diameter. Three angles of attack were considered: $45^{\circ}, 55^{\circ}$, and $65^{\circ}$.

\subsection{Computational Grid}

The quality of the computational grid has a major impact on the computed results. Inadequate grid density and sudden changes in grid point distribution bring about errors in spatial development of vortical structures while discontinuity and non-smoothness in grid line slopes give rise to numerical instabilities due to abrupt variations in grid metrics. Therefore, good quality grid with adequate density is necessary for a successful simulation. The reported results are obtained on a composite O-type grid of size 16.7 million grid points (16.6 million hexahedral cells). There are 510 grid points along the model alone with 201 grid points in the direction normal to the model surface and 121 grid points in the azimuthal direction. Fig. 1 shows the computational domain and the employed grid. The generated grid is smooth and orthogonal. This is essential for the current study as unsteady vortex dominated flow is sensitive to grid quality.

For near-wall regions LES requires axial and azimuthal mesh resolutions of $\Delta x^{+}$and $\Delta \theta^{+} \leq 50$. The superscript ${ }^{+}$indicates nondimensionalization with the friction velocity, 


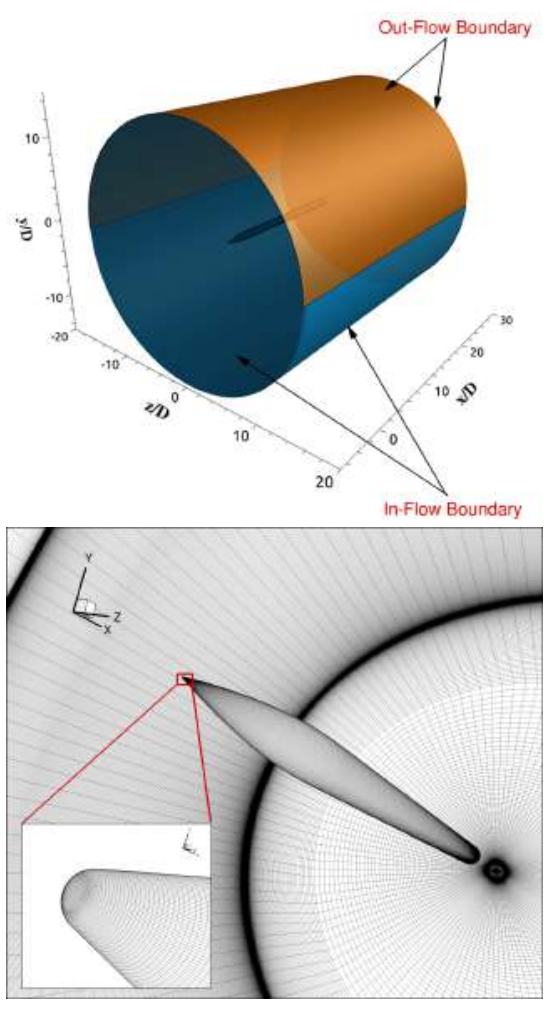

Fig. 1. Computational domain and grid.

$u^{*}$, and $\nu$ (i.e. $\Delta x^{+}=\Delta x u^{*} / \nu$ ). For the current simulation the mesh resolution in the axial direction ranges from $\Delta x^{+}=0.2$, near the nose, to a maximum of 50, 11 diameters downstream from the nose. The resolution in the azimuthal direction is $\Delta \theta^{+}=19$. In the direction normal to the wall, well-resolved LES requires a much finer spacing, $\Delta n^{+} \leq 1$. For the current calculation, the first grid point is located at $\Delta n^{+} \approx 0.35$. Given the operating Reynolds number, the grid resolution is considered sufficient.

\subsection{Dynamic Modes Analysis}

Higher order statistical methods that optimally decompose the flow field have many shortcomings that limit its implementation to turbulent flows. In the last 30 years the most used method is Proper Orthogonal Decomposition (POD) ${ }^{[22,23]}$. Despite the usefulness of POD in analyzing wall bounded and free shear flows, it has many drawbacks. Firstly, the most energetic mode as identified by POD is not always the most important mode in the flow field.
Secondly, POD method is based on an ensemble averaged two-point correlation tensor, therefore, all phase information are lost in the averaging process. Thirdly, the method being based on an ensemble averaged correlation tensor means that it is very expensive to calculate and the statistical error depends on the number of snapshots, i.e., the more snapshots the better the accuracy of the method.

Dynamic Mode Decomposition method, ${ }^{[24,25]}$, does not require any ordering of the data in space or in a form of a matrix, all that matters is a sequence of snapshots in time $V(:, t)$ regardless how they are ordered in space. Hence, DMD method considers a two dimensional matrix for any given flow field whether full three dimensional or plane two dimensional. The first matrix dimension is space and the second is time. Consider a set of data consisting of $n$ snapshots, sampled experimentally or numerically, and ordered in time with a constant time step $\Delta t$ :

$$
\mathbf{V}_{1}^{n}=\left\{v_{1}, v_{2}, v_{3}, \ldots, v_{n}\right\}
$$

The snapshots are assumed to be linearly correlated, i.e., $v_{j}$ is linearly correlated with $v_{j+1}$ or $v_{j+1}=\mathbf{A} v_{j}$, and this linear mapping can be implemented to the whole data set $\mathbf{V}_{1}^{n}$ to obtain a set of the following form:

$$
\left\{v, \mathbf{A} v, \mathbf{A}^{2} v, \ldots \mathbf{A}^{n-1} v\right\}
$$

or

$$
\mathbf{V}_{2}^{n+1}=\mathbf{A V}_{1}^{n}
$$

For sufficiently large sequence, one can assume linear relation between snapshots and construct the $n^{\text {th }}$ snapshot by a linear combination of the preceding $(n+1)$ snapshots, that is:

$$
\mathbf{V}_{2}^{n+1}=\mathbf{A V}_{1}^{n} \approx \mathbf{V}_{1}^{n} \mathbf{S}
$$

$\mathrm{S}$ is a companion matrix that contains the coefficients of the linear mapping. The problem now becomes a least-square problem to find $\mathbf{S}$ that approximate the linear mapping with a minimum error.

$$
[\mathbf{Q}, \mathbf{R}]=\operatorname{qr}\left(\mathbf{V}_{1}^{n-1}\right)
$$




$$
\mathbf{S}=\mathbf{R}^{-1} \mathbf{Q}^{H} \mathbf{V}_{2}^{n}
$$

Once the companion matrix is calculated one can solve the following eigenvalue problem:

$$
\mathrm{S} \Phi=\Gamma \Phi
$$

The eigenvalues of $\mathbf{S}$ contain the growth rates and phase velocities of the modes, while the eigenvectors represent the shape of the dynamic modes.

$$
\lambda_{j}=\frac{\log \left(\boldsymbol{\Gamma}_{j j}\right)}{\Delta t}
$$

The real parts of $\lambda$ represent the growth rates, while the imaginary parts represent the corresponding phase velocities. The dynamic modes are then reconstructed by projecting the original snapshots onto the eigenvectors as follows:

$$
D M(j)=\mathbf{V}_{1}^{n-1}(:,:) \Phi(:, j)
$$

\section{Results}

The calculations were carried out for three angles of attack $\alpha=45^{\circ}, 55^{\circ}$, and $65^{\circ}$. The solution was advanced 25 flow-through times to allow for flow field development. Then a set of 1024 snapshots was collected at ten axial stations. Fig. 2 shows instantaneous pathlines colored by velocity magnitude for the three angles of attack. At $\alpha=45^{\circ}$ the vortex flow is almost symmetric with simultaneous vortex breakdown further downstream. At angle $55^{\circ}$ the flow becomes asymmetric with vortex breakdown moving upstream compared to $\alpha=45^{\circ}$. At $\alpha=65^{\circ}$ the flow is highly asymmetric with asymmetric vortex separation and breakdown starting closer to the nose. Fig. 3 displays top and side views of instantaneous pathlines for the three angles of attack. The figures show an initially symmetric attached vortex flow at $\alpha=45^{\circ}$. As the angle of attack is increased to $\alpha=55^{\circ}$, vortex shedding becomes asymmetric and the vortex flow starts to separate and breaks down. At higher angle, $\alpha=65^{\circ}$, the locations of vortex-separation and
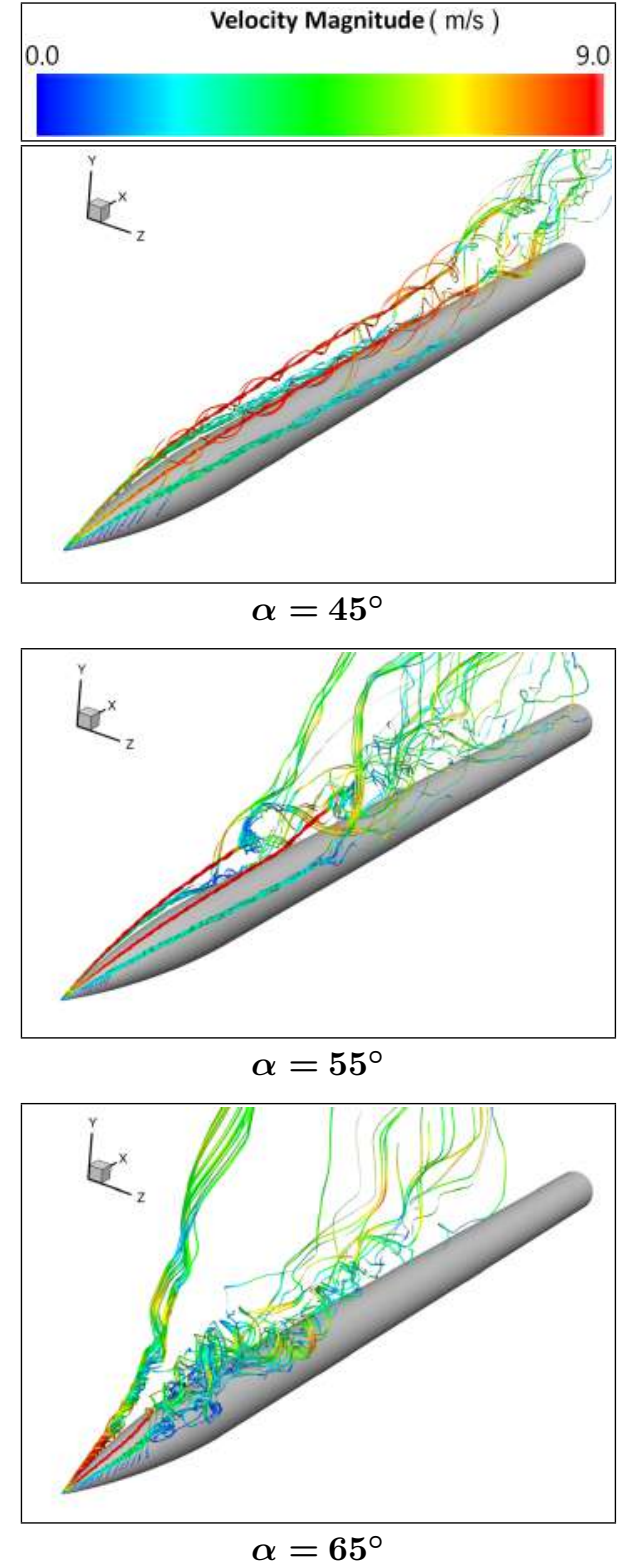

Fig. 2. Instantaneous pathlines at $\alpha=45^{\circ}, 55^{\circ}$, and $65^{\circ}$.

vortex-breakdown propagate upstream and the flow over the body becomes very complex and unsteady. In Fig. 4 instantaneous iso-vorticity surfaces are shown colored by velocity magnitude. The figure reveals the instability in the separated shear layer and the helical shape of the vortex flow with the highest core velocity observed at angle of attack $\alpha=55^{\circ}$. Downstream of the location of vortex breakdown the flow behaves like wake of a 2D cylinder with Karmen vortex shedding. The results confirm 

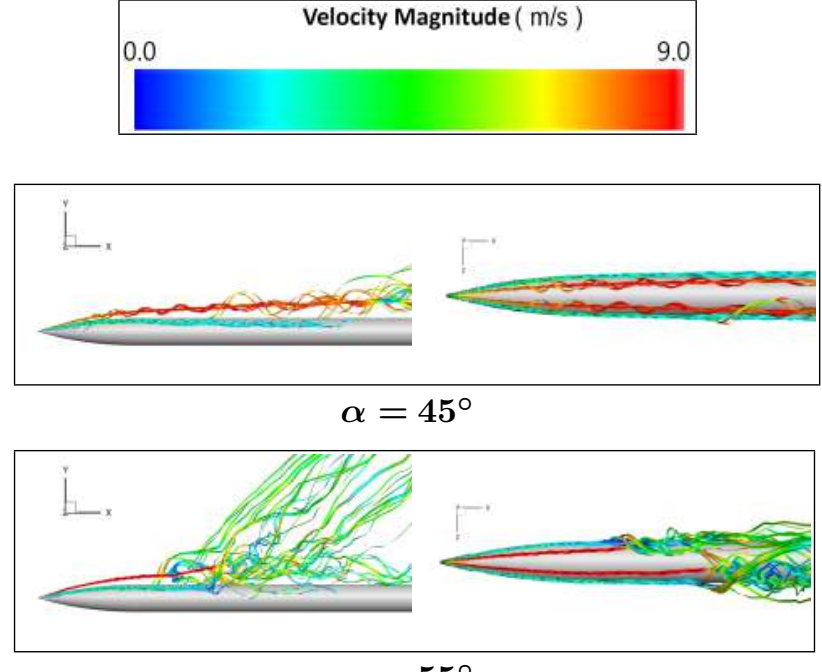

$\alpha=55^{\circ}$

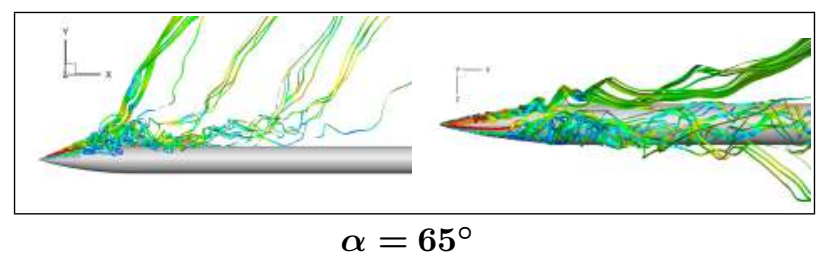

Fig. 3. Top and side views of instantaneous pathlines at $\alpha=45^{\circ}, 55^{\circ}$, and $65^{\circ}$.

the exitance of flow asymmetry in the absence of geometrical imperfections and is most probably due to an absolute instability. It should be emphasized that no artificial asymmetry was introduced in the computations. The computational domain and the grid are symmetric with only double precession roundoff errors. The spatial numerical scheme is a central-difference scheme and the computations were performed using double precession accuracy.

Dynamic Mode Decomposition was applied to the numerical databases that have been generated using large eddy simulation at 10 planes normal to the slender body at several axial locations $(x / \mathrm{D}=0.3$ to 15 as shown in Fig. 5), and at angles of attacks $45^{\circ}, 55^{\circ}$, and $65^{\circ}$. DMD was performed on a sample of instantaneous pressure spanning over five low-frequency cycles ( 1 second). For all 30 planes the plots of real part of the eigenvalues versus imaginary part resulted in a unit circle suggesting that pressure signals are quasiperiodic. DMD analysis identified two distinct
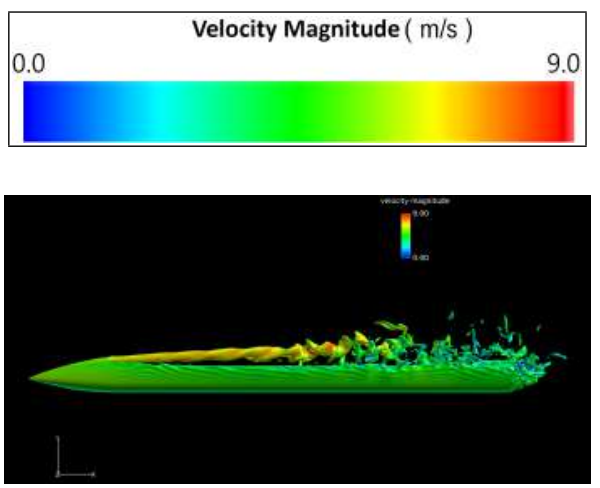

$\alpha=45^{\circ}$

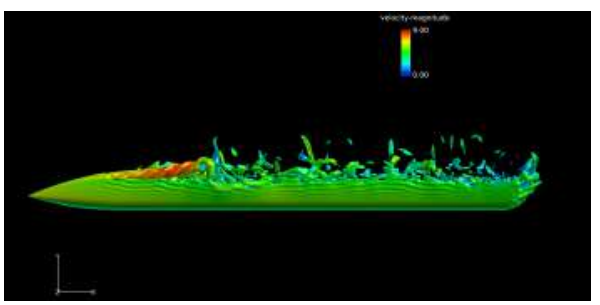

$\alpha=55^{\circ}$

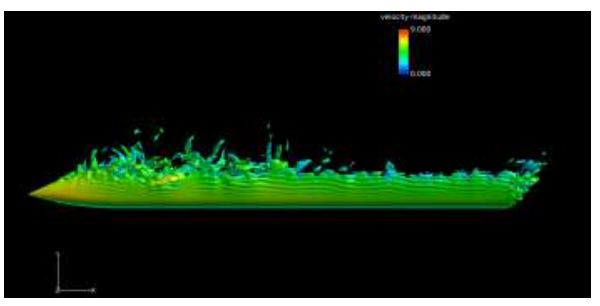

$\alpha=65^{\circ}$

Fig. 4. instantaneous iso-vorticity colored by velocity magnitude at $\alpha=45^{\circ}, 55^{\circ}$, and $65^{\circ}$.

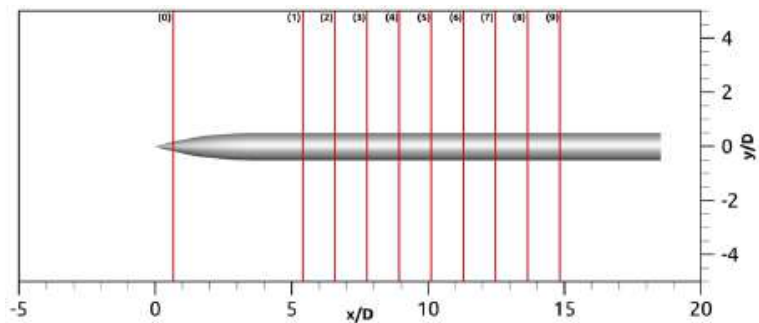

Fig. 5. Axial locations where timeseries data was collected.

unsteady modes; low-frequency wake mode and high-frequency shear layer mode. An extensive search process for the dominant shear layer and dominant wake modes in each downstream plane was carried out. At angle of attack $45^{\circ}$ (Fig. 6) the high-frequency shear mode is dominant at the upstream stations (1) and (2). However, starting from station (3) the dominant mode switches to low-frequency wake mode. 

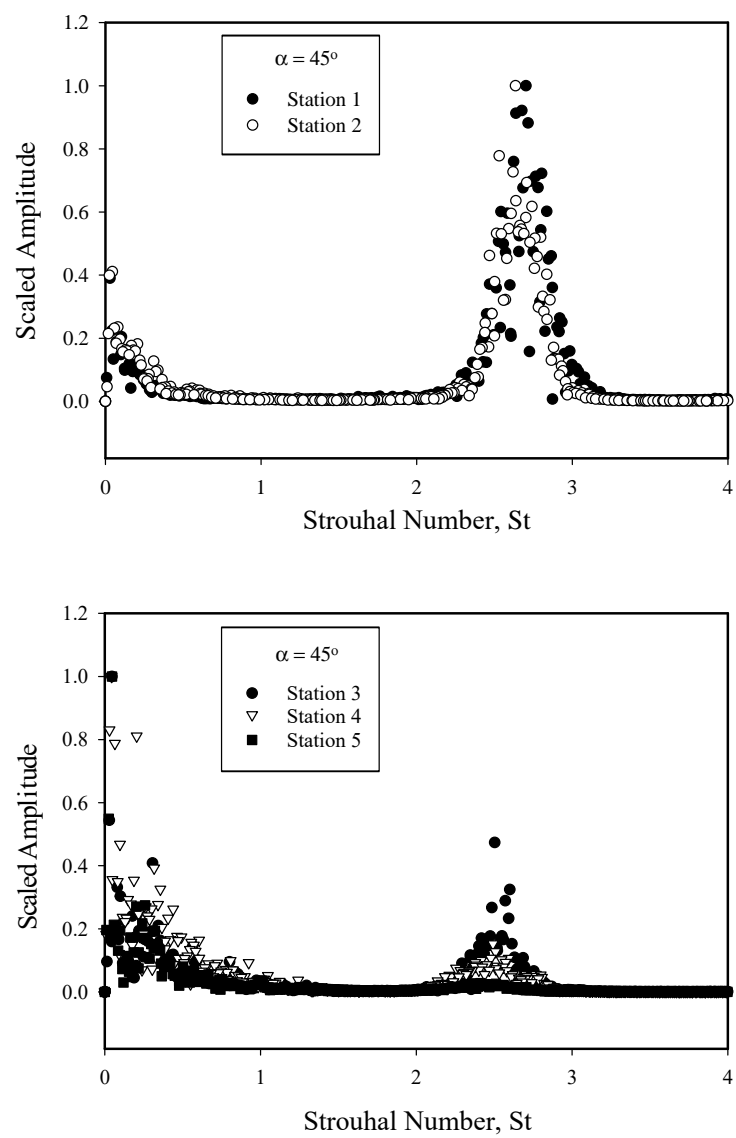

Fig. 6. DMD spectrum for $\alpha=45^{\circ}$ at several axial stations.

For $\alpha=55^{\circ}$ (Fig. 7) no single dominant mode is identified at stations (1), (2), and (3). However, a broadband of low frequencies is observed. At stations (4) and (5) the high-frequency shearlayer is clearly dominant. At $\alpha=65^{\circ}$ (Fig. 8) the spectrum shows a wide range of modes indicating vortex breakdown and transition wake flow. Further investigations are necessary to understand the relation between mode switching and the initiation of flow asymmetry as the angle of attack is increased.

To investigate the type of the detected dynamic modes, a reconstruction of the modes is performed at station (2) for $\alpha=45^{\circ}$. Fig. 9 and Fig. 10 show the low-frequency and high-frequency dynamic modes, reconstructed by projecting the instantaneous data onto the modes' eigenfunctions. The projections show that the low-frequency mode is as-
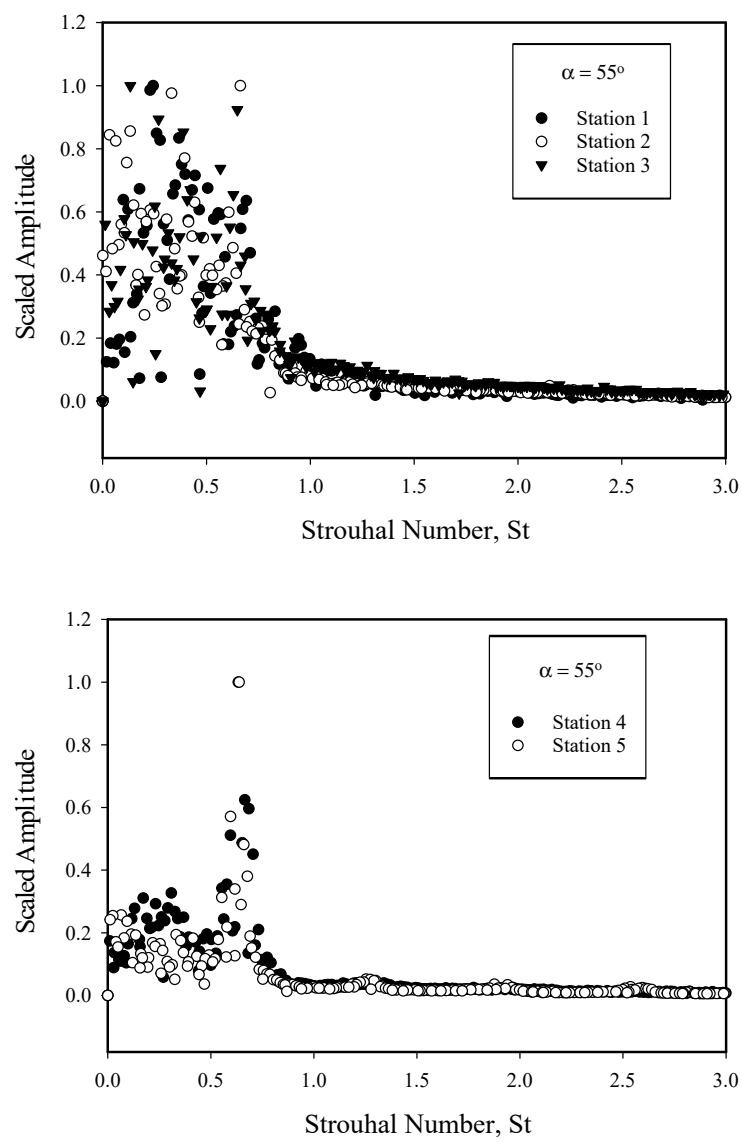

Fig. 7. DMD spectrum for $\alpha=55^{\circ}$ at several axial stations.

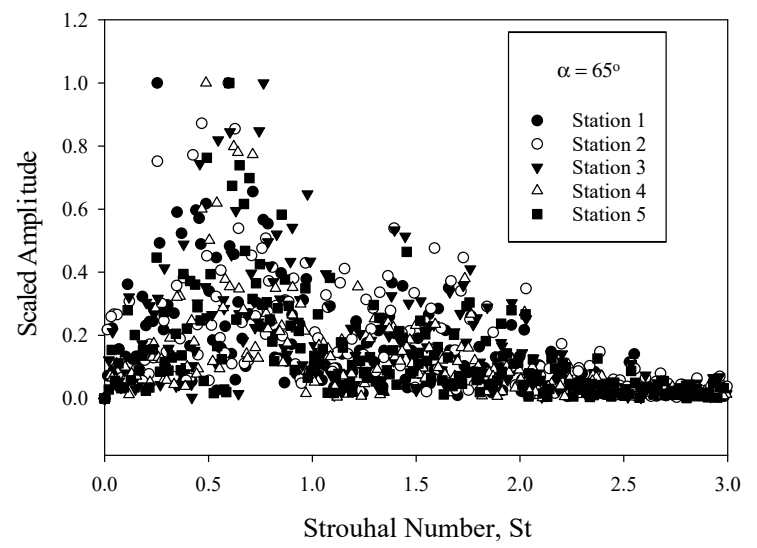

Fig. 8. DMD spectrum for $\alpha=65^{\circ}$ at several axial stations.

sociated with the unsteady wake and the highfrequency mode is associated with unsteady shear layer which is in agreement with experimental observations of Degani and Zilliac ${ }^{[10]}$. 


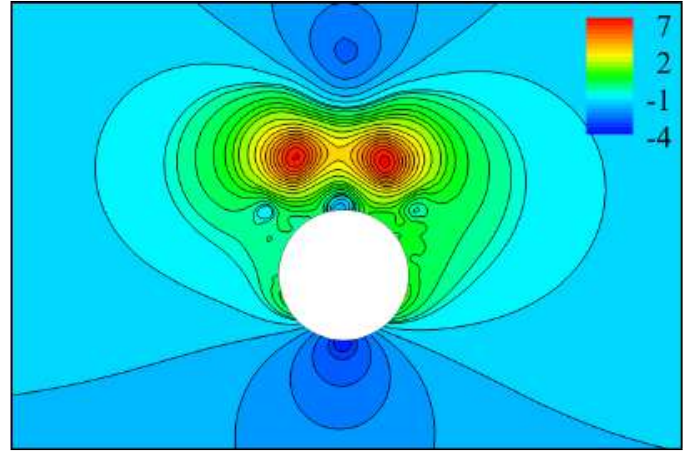

Fig. 9. Reconstructed low frequency mode at station (2), $\alpha=45^{\circ}$.

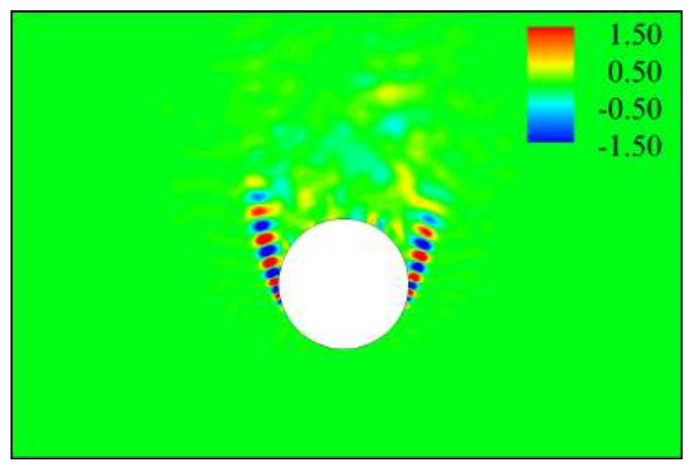

Fig. 10. Reconstructed high frequency mode at station (2), $\alpha=45^{\circ}$.

\section{Summary}

LES of the flow around an ogive-cylinder body at high angles of attack have been presented. The asymmetric vortex flow phenomenon was observed at angles of attack of $\alpha=55^{\circ}$ and $\alpha=65^{\circ}$. The results also showed that the phenomenon is present in the absence of artificial geometrical or flow asymmetry. It is concluded that flow asymmetry is due to an absolute instability rather than a convective instability. An investigation of the unsteady flow field was carried out using dynamic mode decomposition. The analysis identified two distinct unsteady modes; unsteady wake and unsteady shear layer. At angle of attack $45^{\circ}$ the shear mode is dominant upstream and the lowfrequency wake mode is dominant downstream. For $\alpha=55^{\circ}$ the high-frequency shear-layer is dominant downstream of vortex breakdown. At $\alpha=65^{\circ}$ the spectrum shows a wide range of modes. Further investigations are necessary to understand the relation between mode switching and the initiation of flow asymmetry as the angle of attack is increased.

\section{Acknowledgements}

This work was supported by (KACST) under the NSTIP program - Project. No. (08SPA135-03). All computations were performed on Aziz Supercomputer at King Abdulaziz university's High Performance Computing Center (http://hpc.kau.edu.sa/). The authors acknowledge the computer time and technical support provided by the center.

\section{References}

[1] Zilliac, G.G., Degani, D., and Tobak, M., Asymmetric vortices on a slender body of revolution. AIAA Journal 29(5), 667-675 (1991). DOI 10.2514/3.59934

[2] Gowen, F. E., and Perkins, E. W., A study of the effects of body shape on the vortex wakes of inclined bodies at a mach number of 2. Tech. Rep. NACA-RM-A53I17, NACA (1953).

[3] Wardlaw, A.B., and Morrison, A. M., Induced side forces at high angles of attack. Journal of Spacecraft and Rockets 13(10), 589-593 (1976). DOI 10.2514/3.27931

[4] Leu, T.-S., Chang, J.-R., and Lu, P.-J., Experimental investigation of side force control on cone-cylinder slender bodies with flexible micro balloon actuators. Experimental Thermal and Fluid Science 29(8), 909-918 (2005). DOI 10.1016/j.expthermflusci. 2004.12.005

[5] Banks, D. W., Fisher, D. F., Hall, R. M., Erickson, G. E., Murri, D. G.., Grafton, S. B., and Sewall, W. G., The F/A-18 high-angle-of-attack ground-to-flight correlation: lessons learned. Tech. Rep. NASA-TM-4783, NASA (1997).

[6] Bridges, D. H., The asymmetric vortex wake problemasking the right question. In: The $36^{\text {th }}$ AIAA Fluid Dynamics Conference, AIAA 2006-3553, pp. 1737-1765 (2006). DOI 10.2514/ $6.2006-3553$

[7] Stahl, W, and Asghar, A, Dependence on reynolds number of onset of vortex asymmetry behind circular cone. In: $34^{\text {th }}$ Aerospace Sciences Meeting and Exhibit, AIAA 1996-0064 (1996). DOI 10.2514/6.1996-64

[8] Liu, P., and Deng, X., Experimental investigation of aerodynamic characteristics on slender bodies at high angles of attack. Canadian Aeronautics and Space Journal 49(1), 31-40 (2003). DOI 10.5589/q03-002

[9] Xueying, D., and Yankui, W., Asymmetric vortices flow over slender body and its active control at high angle of attack. Acta Mechanica Sinica 20(6), 567-579 (2004). DOI 10.1007/BF02485860

[10] Degani, D., and Zilliac, G.G., Experimental study of nonsteady asymmetric flow around an ogive-cylinder at incidence. AIAA Journal 28(4), 642-649 (1990). DOI 10.2514/3.10441 
[11] Ma, B., Huang, Yu, and Liu, T., Low-frequency unsteadiness of vortex wakes over slender bodies at high angle of attack. Chinese Journal of Aeronautics 27(4), 772-780 (2014). DOI 10.1016/j. cja.2014.06.011

[12] Liu, CH, Wong, T.-C., and Kandil, O. A., Prediction of asymmetric vortical flows around slender bodies using navier-stokes equations. Fluid Dynamics Research 10(4-6), 409 (1992). DOI 10.1016/0169-5983(92)90032-r

[13] Degani, D., Instabilities of flows over bodies at large incidence. AIAA Journal 30(1), 94-100 (1992). DOI 10.2514/3.10887

[14] Degani, D., and Levy, Y., Asymmetric turbulent vortical flows over slender bodies. AIAA Journal 30(9), 2267-2273 (1992). DOI $10.2514 / 3.11214$

[15] Levy, Y., Hesselnik, L., and Degani, D., Systematic study of the correlation between geometrical disturbances and flow asymmetries. AIAA Journal 34(4), 772-777 (1996). DOI 10.2514/3.13139

[16] Murman, S. M., Geometric perturbations and asymmetric vortex shedding about slender pointed bodies. AIAA Journal pp. 20004103 (2000). DOI 10.2514/6.2000-4103

[17] Kandil, O. A., Wong, T.-C., and Liu, CH, Navier-stokes computations of symmetric and asymmetric vortex shedding around slender bodies. AIAA Journal pp. 89-3397 (1989). DOI 10.2514/6.1989-3397

[18] Thomson, K.D., and Morrison, D.F., The spacing, position and strength of vortices in the wake of slender cylindrical bodies at large incidence. Journal of Fluid Mechanics 50(04), 751-783 (1971). DOI 10.1017/s0022112071002878
[19] Degani, D., and Marcus, S.W., Thin vs full navier-stokes computation for high-angle-of-attack aerodynamics. AIAA Journal 35(3), 565-567 (1997). DOI 10.2514/2.134

[20] Germano, M., Piomelli, U., Moin, P., and Cabot, W. H., A dynamic subgrid-scale eddy viscosity model. Phys. Fluids A-Fluid 3(7), 1760-1765 (1991). DOI 10.1063/1.857955

[21] Lilly, D.K., A proposed modification of the germano subgridscale closure method. Phys. Fluids A-Fluid 4(3), 633-635 (1992). DOI 10.1063/1.858280

[22] Lumley, J. L., The structure of inhomogeneous turbulent flows. In: Atmospheric Turbulence and Radio Wave Propagation, pp. 166-178. Moscow (1967).

[23] Holmes, P., Lumley, J. L., and Berkooz, G., Turbulence, Coherent Structures, Dynamical Systems and Symmetry. Cambridge University Press (1996). DOI 10.1017/cbo9780511622700

[24] Rowley,C. W., Mezic,I., Bagheri,S., Schlatter,P., and Henningson,D. S., Spectral analysis of nonlinear flows. Journal of Fluid Mechanics 641, 115-127 (2009). DOI 10.1017/ S0022112009992059

[25] Schmid,P. J., Dynamic mode decomposition of numerical and experimental data. Journal of Fluid Mechanics 656, 5-28 (2010). DOI 10.1017/S0022112010001217 


\section{دراسة التدفق حول جسم أسطواني عند زوايا الهجوم العالية إبراهيم محمدالخضر القاضي و الطيب محمد الجاك}

قسم هندسة الطيران ، كلية الهندسة، جامعة الملك عبدالعزيز، جدة، المملكة العربية السعودية ialqadi@kau.edu.sa

الدستخلص. يقدم هذا البحث دراسة عددية للتدفق حول جسم أسطواني عند زوايا هجوم عالية، وقد تم إجراء الحسابات بطريقة "محاكاة الدوامة الكبيرة"، ولوحظ وجود تدفق دوامات غير متماثل عند زوايا

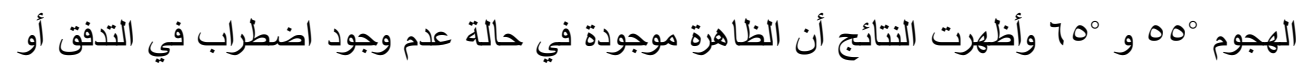
عدم تماثل في الجسم الأسطواني، وخلافا للفكرة المقبولة والتي تثير إلى أن عدم تناسق التدفق يرجع

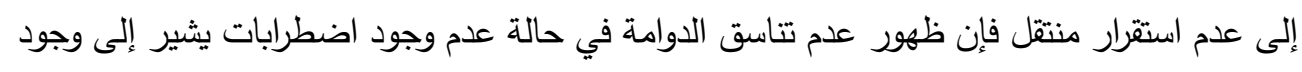

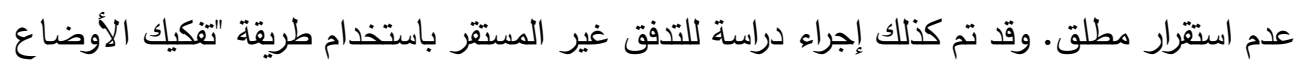

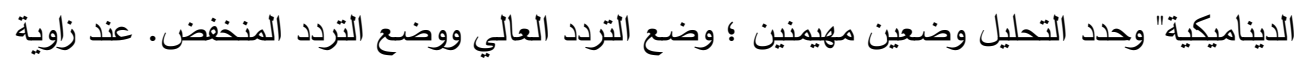

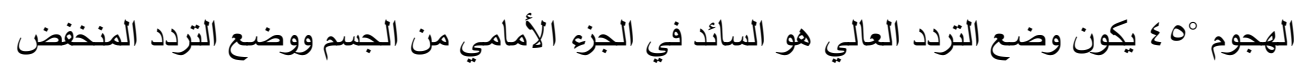

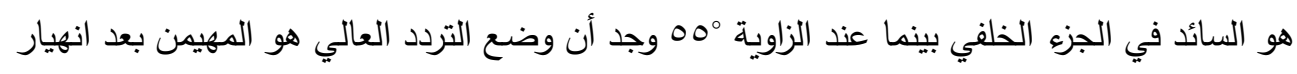

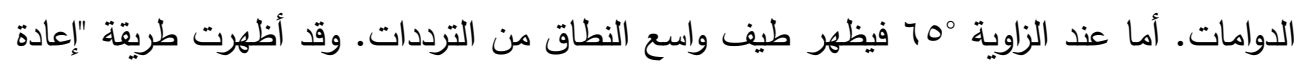
بناء الأوضاع الديناميكية" أن وضع التردد المنخفض يرتبط بالدوامات غير المستقرة وأن وضع التردد العالي يرتبط بطبقة القص المنفصلة غير المستقرة. 
